\title{
New numerical evaluation of parallelepiped Nal(TI) detector geometrical and total efficiencies using extended sources
}

\author{
S.F. Noureddeen ${ }^{1}$ and M.I. Abbas ${ }^{2, *}$ \\ ${ }^{1}$ Physics Department, Faculty of Science, Lebanese University, Beirut, Lebanon. \\ 2 Physics Department, Faculty of Science, Alexandria University, 21511 Alexandria, Egypt.
}

Received 21 November 2016 / Accepted 1 August 2017

\begin{abstract}
Gamma-ray measurements in terrestrial/environmental samples require the use of high efficient detectors because of the low level of the radionuclide activity concentrations in the samples; thus scintillators are suitable for this purpose. The design of $4 \pi \mathrm{NaI}(\mathrm{Tl})$ parallelepiped $\gamma$-ray scintillation detector with central square left void is considered an instructive case in radiation measurements, where it can be easily used in several critical applications including medical therapy, radiation protection, environmental exposure monitoring and neutron interaction. It is also extremely important to measure the activities of low radioactivity samples. Therefore, the understanding of the full scope of the multidimensional calibration and measurement process must be done with high accuracy. The present work is essentially concerned in introducing a new numerical evaluation to calculate the geometrical and total efficiency of a $4 \pi \mathrm{NaI}(\mathrm{Tl})$ parallelepiped scintillator detector using isotropic radiating $\gamma$-ray point source. Also the average path lengths traveled by the photon inside the detector active medium have been calculated in order to study the characteristics of the source-to-detector configurations. This method depends mainly on the calculation of the solid angle subtended to the radioactive point source situated at various positions inside the detector and the photon path lengths through the detector active medium. In addition, the attenuation of the stainless steel absorber between the source and the detector material has been studied. The obtained results were compared with Monte Carlo and experimental published data to show the method's validity in the calibration process.
\end{abstract}

Keywords: parallelepiped $\mathrm{NaI}(\mathrm{Tl})$ detector / total efficiency / numerical analytical method

\section{Introduction}

In gamma-ray spectroscopy using HPGe or $\mathrm{NaI}(\mathrm{Tl})$ detectors of any geometrical shape, one usually needs to know the geometrical, total and the full-energy peak efficiencies for any specific source-detector configuration of concern. Because the experimental work is difficult for extended sources, many researches have been focused on the development of computational techniques to determine these efficiencies. There are three common methods used in this field, the semi-empirical, the Monte Carlo, and the direct mathematical methods. The semi-empirical method has been used by several authors (Sánchez-Reyes et al., 1987; Jiang et al., 1998; Kis et al., 1998; Chatani, 1999). In this method, the full-energy peak efficiency is performed at discrete energies so one has to fit a curve using the measured points. The second method statistically models the interactions of each

\footnotetext{
*Corresponding author: mabbas@physicist .net; mahmoud.abbas@alexu.edu.eg
}

photon emitted from the source until it leaves the detector or deposit all of its energy within the active detector volume (Cejnar and Kováŕ, 1980; Baba et al., 1991; Yücel et al., 1996). For this method to be exact, one needs to model sufficiently a large number of source particles and details about the composition of detector and intercepting layers. There are no approximations or limitations to the source-detector configurations, but it is computationally time consuming.

Presently, $\mathrm{NaI}(\mathrm{Tl})$ detectors have been commonly used in different detecting systems in the form of well-type, parallelepiped, cylindrical, hexagonal, borehole, sphere, etc., where it has relative simplicity, low cost of crystal preparation, high mass number, high efficiency and room temperature operation (Al-Arbawy, 2014). The $4 \pi \mathrm{NaI}(\mathrm{Tl})$ parallelepiped $\gamma$-ray scintillator detector is made up of eight arrays with central square left void as special design has a broad solid angle $(3.83 \pi \mathrm{sr})$ subtended to the isotropic radiating $\gamma$-ray point sources, if it is used for the calibration process (Byun et al., 2004). The aim of this work is to apply the new numerical analytical method (NAM) to calculate the average path length and the total efficiency of this detector. This can be done by using different radiating point sources of low energies 
placed inside the detector. In addition, the total efficiency is determined for disk and cylindrical sources of different energies. These sources are placed at successive distances inside the detector. This method is an extension of the previous work for a new detector type and geometry (Abbas, 2001a, 2001b, 2006; Nafee et al., 2010; Badawi et al., 2013; Gouda et al., 2015). All the analytical integrations were solved numerically by using the trapezoidal rule. The arrangement of this paper is as follows: Section 2 presents direct mathematical formulae for calculating the total efficiency in the case of isotopically radiating axial point, non-axial point, disk and volumetric (cylindrical) sources. The attenuation of photons by the source container and the detector housing materials is taken into account. Section 3 compares the results of this approach and published data. Results show the validity of the recent approach. Conclusions are presented in Section 4.

\section{Mathematical viewpoint}

This section gives an explanation about the new numerical analytical method (NAM) based on the direct mathematical method (Abbas, 2001a, 2001b, 2006; Nafee et al., 2010; Abbas and Noureddeen, 2011; Badawi et al., 2013; Gouda et al., 2015). The method will be useful to find the geometrical efficiency, average path length and total efficiency of the $4 \pi \mathrm{NaI}(\mathrm{Tl})$ parallelepiped $(a \times b \times c)$ where $a=b=30.6 \mathrm{~cm}$ and $c=40.6 \mathrm{~cm}$ (Byun et al., 2004). The central squared void part has the following sizes $a_{1}=b_{1}=10.2 \mathrm{~cm}$. The housing of the $\mathrm{NaI}(\mathrm{Tl})$ crystal is $1 \mathrm{~mm}$ thick stainless steel as shown in Figure 1. The calibration source can be placed in the center of the square hole of the parallelepiped detector (see Fig. 1) or at distance $\rho$ from the center (see Fig. 2a). The absolute efficiency $\varepsilon$ for any specified source-to-detector configuration can be given by:

$$
\varepsilon=\frac{1}{4 . \pi} \int_{\phi \theta}\left(1-e^{-\mu d}\right) \sin (\theta) d \theta d \phi
$$

The polar angle $\theta$ and the azimuthal angle $\varphi$ control the direction of the incident photons; $\mu$ is the attenuation coefficient of the detector material without the coherent part (National Institute of Standard and Technology, Physical Measurements Laboratory, 1998); $d$ is the distance covered by the photon inside the detector active medium.

\subsection{The case of an axial point source placed inside the detector square hole}

In this case, the total efficiency $\varepsilon_{t}$ is calculated by the spherical coordinate system and is given by equation (2):

$$
\begin{aligned}
\varepsilon_{t(\text { point }- \text { Axial })} & =\frac{8}{4 . \pi} \int_{0}^{\phi_{1} \theta_{2}} \int_{\theta_{1}} \sin \theta d \theta d \phi \\
& +\frac{8}{4 . \pi} \int_{0}^{\phi_{1} \frac{\pi}{2}} \int_{\theta_{2}} f_{2} \sin \theta d \theta d \phi,
\end{aligned}
$$

where $\theta_{1}$ and $\theta_{2}$ are the polar angles that allow the photons to go into the detector active medium in case of an axial isotropic

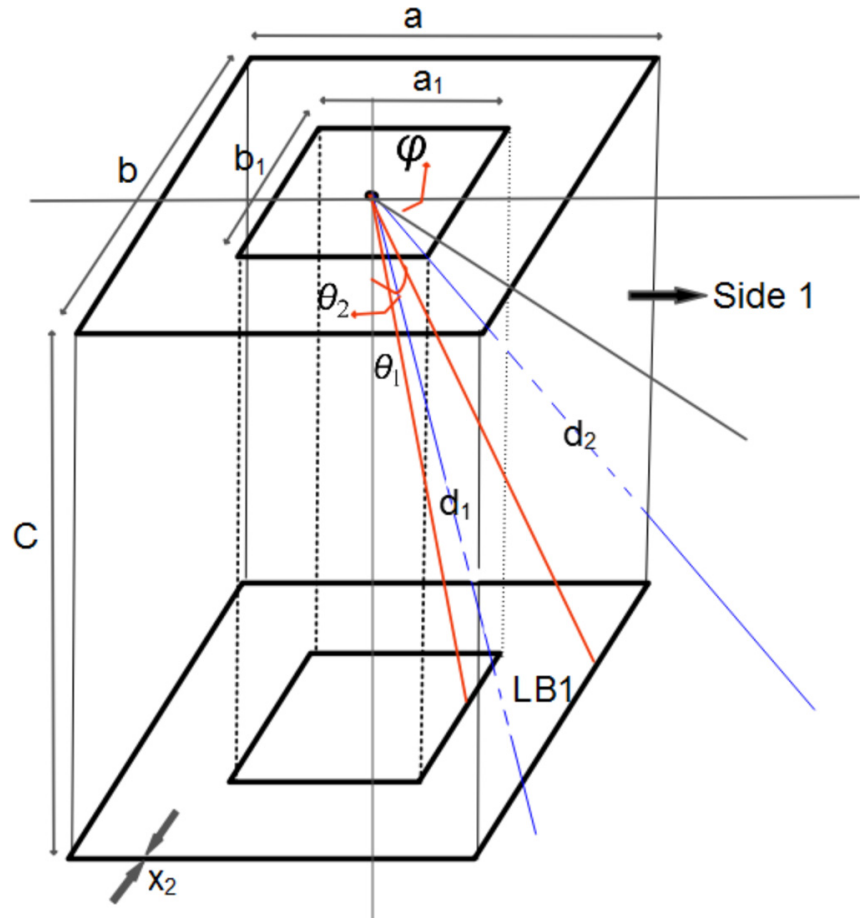

Fig. 1. The point source is situated at the parallelepiped detector major axis.

radiating point source:

$$
\begin{aligned}
& \theta_{1}=\tan ^{-1}\left(\frac{a_{1}}{2 c}\right), \\
& \theta_{2}=\tan ^{-1}\left(\frac{a}{2 c}\right) .
\end{aligned}
$$

The azimuthal angle $\varphi$ is given by:

$$
\varphi_{1}=\tan ^{-1}\left(\frac{b}{a}\right) \text {. }
$$

The partial efficiency factor $f_{i}$ is given by:

$$
f_{i}=f_{\text {att }}\left(1-e^{-\mu \cdot d_{i}}\right), i=1,2,
$$

$d_{i}$ are the possible path lengths traveled by the photon within the detector active volume as shown in Figure 1. So to calculate the path length there are two main cases to be considered.

The striking photon may enter the detector inner side and emerge from its lower base layer (LB1).

$$
d_{1}=\frac{c}{\cos (\theta)}-\frac{a}{2 \sin (\theta) \cos (\phi)} \text {. }
$$

The striking photon may enter the detector inner side and emerge from its face layer (side 1).

$$
d_{2}=\frac{\left(a-a_{1}\right)}{2 \sin (\theta) \cos (\phi)} .
$$



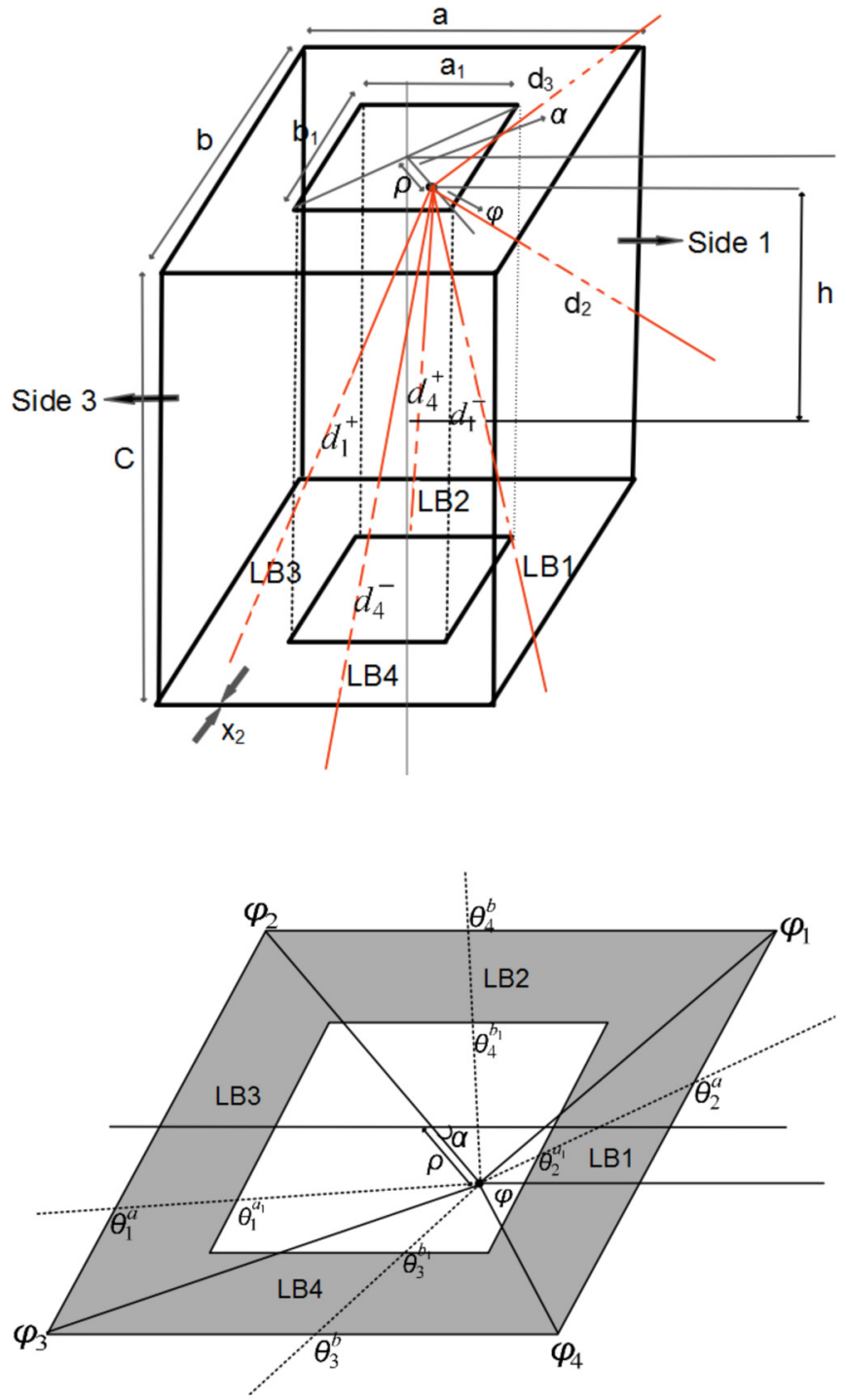

Fig. 2. (a) Possible path lengths through detector active medium for non-axial point source $P(\rho, h)$ placed inside the detector, (b) possible polar and azimuthal angles for non-axial point source $P(\rho, h)$ placed inside the detector.

The attenuation factor, $f_{a t t}$, determining the photon attenuation by the source container and the stainless steel detector housing materials, is expressed as:

$$
f_{\text {att }}=e^{-\frac{\left(\mu_{1} x_{1}+\mu_{2} \cdot x_{2}\right)}{\sin (\theta)}}
$$

$\mu_{1}$ and $x_{1}$ are the attenuation coefficient and thickness of the source container; $\mu_{2}$ and $x_{2}$ are the attenuation coefficient and thickness of the stainless-steel detector housing.

The average path length $\bar{d}$ traveled by the photon inside the parallelepiped $\mathrm{NaI}(\mathrm{Tl})$ detector material depends on the position of the incident photon and the solid angle $\Omega$, and is given by:

$$
\bar{d}=\frac{\int_{\Omega} d(\theta, \varphi) d \Omega}{\int_{\Omega} d \Omega}=\frac{\iint_{\varphi} d(\theta, \varphi) \sin \theta d \theta d \varphi}{\Omega},
$$

$$
\bar{d}=\frac{\int_{0}^{\phi_{1} \theta_{2}} \int_{\theta_{1}} d_{1} \sin \theta d \theta d \phi+\int_{0}^{\phi_{1} \frac{\pi}{2}} \int_{\theta_{2}} d_{2} \sin \theta d \theta d \phi}{\int_{0}^{\phi_{1} \theta_{2}} \int_{\theta_{1}} \sin \theta d \theta d \phi+\int_{0}^{\phi_{1}} \int_{\theta_{2}}^{\frac{\pi}{2}} \sin \theta d \theta d \phi} .
$$

\subsection{The case of non-axial point source placed inside the detector square hole}

In the case of an arbitrarily positioned isotropic radiating non-axial point source $P(\rho, h)$, see Figure 2, placed inside the void part the total efficiency is given by:

$$
\varepsilon_{t(\text { point-nonaxial })}=\sum_{n=1}^{5} J_{n},
$$

where

$$
\begin{gathered}
J_{1}=\int_{\phi_{2}}^{\phi_{3}} \int_{\theta_{1}^{a 1}}^{\theta_{1}^{a}} f_{1}^{+} d \theta d \phi+\int_{\phi_{2}}^{\phi_{3}} \int_{\theta_{1}^{a}}^{\frac{\pi}{2}} f_{2} d \theta d \phi, \\
J_{2}=\int_{0}^{\phi_{1}} \int_{\theta_{2}^{a 1}}^{\theta_{2}^{a}} f_{1}^{-} d \theta d \phi+\int_{0}^{\phi_{1}} \int_{\theta_{2}^{a}}^{\frac{\pi}{2}} f_{2} d \theta d \phi, \\
J_{3}=\int_{\phi_{4}}^{2 \pi} \int_{\theta_{2}^{a 1}}^{\theta_{2}^{a}} f_{1}^{-} d \theta d \phi+\int_{\phi_{4}}^{2 \pi} \int_{\theta_{2}^{a}}^{\frac{\pi}{2}} f_{2} d \theta d \phi, \\
J_{4}=\int_{\phi_{1}}^{\phi_{2}} \int_{\theta_{3}^{b}}^{\frac{\pi}{2}} f_{3} d \theta d \phi+\int_{\phi_{1}}^{\phi_{2}} \int_{\theta_{3}^{b_{1}}}^{\theta_{3}^{b}} f_{4}^{+} d \theta d \phi, \\
J_{5}=\int_{\phi_{3}}^{\phi_{4}} \int_{\theta_{4}^{b_{1}}}^{\theta_{3}^{b}} f_{3} d \theta d \phi+\int_{\phi_{3}}^{\phi_{4}} \int_{\theta_{3}^{b}}^{\frac{\pi}{2}} f_{4}^{-} d \theta d \phi, \\
f_{i}^{ \pm}=\left(1-e^{-\mu \cdot d_{i}^{ \pm}}\right) \cdot \sin (\theta),
\end{gathered}
$$

$d_{i}^{ \pm}$represents the photon path lengths traveled through the detector active volume and $i=1,2$. There are four main cases for each $d$ to be considered:

- the striking photon may enter by the detector inner side and emerge from its left (LB3) or right base layer (LB1);

$$
d_{1}^{ \pm}=\frac{c}{\cos (\theta)}-\frac{a_{1} \pm 2 \rho \cos (\alpha)}{2 \sin (\theta) \cos (\phi)}
$$

- the striking photon may enter by the detector inner side and emerge from its outer left (side 3) or right side (side 1);

$$
d_{2}=\frac{a-a_{1}}{2 \sin (\theta) \cos (\phi)}
$$

- the striking photon may enter by the detector inner side and emerge from its outer front (above lower base 4 LB4) or back side (above lower base 3 LB2); 


$$
d_{3}=\frac{b-b_{1}}{2 \sin (\theta) \cos (\phi)}
$$

- the striking photon may enter by the detector inner side and emerge from its outer (LB4) or inner base (LB2);

$$
d_{4}^{ \pm}=\frac{c}{\cos (\theta)}-\frac{b_{1}}{2 \sin (\theta) \cos (\phi)} \pm \frac{\rho \sin (\alpha)}{\sin (\theta) \sin (\phi)} .
$$

Finally, the values of the polar $\theta$ and the azimuthal $\varphi$ angles are given by:

$$
\begin{aligned}
\theta_{1}^{a, a_{1}} & =\tan ^{-1}\left(\frac{a, a_{1}+2 \rho \cos (\alpha)}{2 c \cos (\phi)}\right), \\
\theta_{2}^{a, a_{1}} & =\tan ^{-1}\left(\frac{a, a_{1}-2 \rho \cos (\alpha)}{2 c \cos (\phi)}\right), \\
\theta_{3}^{b, b_{1}} & =\tan ^{-1}\left(\frac{b, b_{1}-2 \rho \sin (\alpha)}{2 c \sin (\phi)}\right), \\
\theta_{4}^{b, b_{1}} & =\tan ^{-1}\left(\frac{b, b_{1}+2 \rho \sin (\alpha)}{2 c \sin (\phi)}\right), \\
\phi_{1} & =\tan ^{-1}\left(\frac{\frac{a}{2}-\rho \sin (\alpha)}{\frac{b}{2}-\rho \cos (\alpha)}\right), \\
\phi_{2} & =\frac{\pi}{2}+\tan ^{-1}\left(\frac{\frac{b}{2}-\rho \cos (\alpha)}{\frac{a}{2}-\rho \sin (\alpha)}\right), \\
\phi_{3}= & \frac{3 \pi}{2}+\tan ^{-1}\left(\frac{\frac{a}{2}-\rho \cos (\alpha)}{\frac{b}{2}-\rho \sin (\alpha)}\right) . \tan ^{-1}\left(\frac{\frac{a}{2}+\rho \sin (\alpha)}{\frac{b}{2}+\rho \cos (\alpha)}\right),
\end{aligned}
$$

\subsection{The case of a concentric disk source placed inside the detector hole}

The efficiency of a $4 \pi \mathrm{NaI}(\mathrm{Tl})(a \times b \times c)$ detector, with a central rectangle hole $\left(a_{1} \times b_{1} \times c\right)$, arising from a thin disk source of radius $s$, is given by:

$$
\varepsilon_{t(\text { disk })}=\frac{2}{s^{2}} \int_{0}^{s} \varepsilon_{t(\text { point-nonaxial })} \rho d \rho,
$$

where $\varepsilon_{t(\text { point-nonaxial) }}$ is the total efficiency of a non-axial point source, as identified before in equation (12).

\subsection{The case of a concentric cylindrical source placed inside the detector hole}

The efficiency of a $4 \pi \mathrm{NaI}(\mathrm{Tl})(a \times b \times c)$ detector, with a central rectangle hole $\left(a_{1} \times b_{1} \times c\right)$, arising from a cylindrical source with radius $s$ and height $\left(h_{2}-h_{1}\right)$, is given by:

$$
\varepsilon_{t(\text { cylindrical })}=\frac{1}{h_{2}-h_{1}} \int_{0}^{h} \varepsilon_{t(\text { disk })} d h,
$$

where $\epsilon_{t(\text { disk })}$ is the total efficiency of a disk source, as identified before in equation (31)

\section{Results}

The new design of the parallelepiped $\gamma$-ray detector shape presents a great interest in studying the absolute efficiencies. According to Byun et al. (2004) the total and full-energy peak efficiencies of the parallelepiped $\mathrm{NaI}(\mathrm{Tl})$ detector were measured by allowing a beam of photons from an isotropic radiating $\gamma$-ray point and cubic sources to enter the detector. The measurements were performed by using a parallelepiped $\mathrm{NaI}(\mathrm{Tl})\left(30.6 \times 30.6 \times 40.6 \mathrm{~cm}^{3}\right)$ with a void central rectangular hole of $\left(10.2 \times 10.2 \mathrm{~cm}^{2}\right)$. The housing of the $\mathrm{NaI}(\mathrm{Tl})$ crystal is $1 \mathrm{~mm}$ thick stainless steel. The point sources of different energies were positioned axially at the center of the detector. These measured and simulated efficiencies (Byun et al., 2004) were summarized in Table 1 and compared with the direct theoretical efficiencies from the present work. Also, comparisons between simulated and theoretical relative total efficiencies (present work) at different heights from the center of the detector for $0.511 \mathrm{MeV}$ were summarized in Table 2 . The numerical evaluation of the integrals is performed using the MathCad 14.0 software. The present values of the calculated efficiencies are much better than that of M.C. (Byun et al., 2004) and, in particular, our values are obtained without any adjustment of the detector geometrical parameters.

The deviation percentage, $\Delta_{1} \%$ and $\Delta_{2} \%$ between the [ $\varepsilon_{\text {Calculated }} / \varepsilon_{\text {Measured }}$ and $\varepsilon_{\text {Calculated }} / \varepsilon_{\text {Simulated }}$ is based on the following equations, respectively:

$$
\begin{aligned}
& \Delta_{1} \%=\frac{\varepsilon_{\text {calculated }}-\varepsilon_{\text {measured }}}{\varepsilon_{\text {calculated }}} \times 100, \\
& \Delta_{2} \%=\frac{\varepsilon_{\text {calculated }}-\varepsilon_{\text {simulated }}}{\varepsilon_{\text {calculated }}} \times 100,
\end{aligned}
$$

where $\varepsilon_{\text {calculated }}$ is the calculated efficiency using the present work, $\varepsilon_{\text {simulated }}$ is the calculated efficiency using Monte Carlo (Byun et al., 2004) and $\varepsilon_{\text {measured }}$ is the measured efficiency experimentally (Byun et al., 2004).

In addition, the total efficiency is plotted at different energies and the graph is shown in Figure 3. The graph shows that the total efficiency decreased by $12 \%$ in the region between 0.5 and $3 \mathrm{MeV}$. This decrease is related to the dependence of the efficiency on the attenuation coefficient as given by equation (2). Also, the total efficiency decreased more significantly in the lower-energy region due to absorption of gamma rays by stainless steel. 
Table 1. Comparison between calculated (present work), experimental (Byun et al., 2004) and simulated (Byun et al., 2004) total efficiencies for an isotropic radiating axial point sources placed at the center of a $4 \pi \mathrm{NaI}(\mathrm{Tl})\left(30.6 \times 30.6 \times 40.6 \mathrm{~cm}^{3}\right)$ detector with a central rectangle hole $\left(10.2 \times 10.2 \times 40.6 \mathrm{~cm}^{3}\right)$.

\begin{tabular}{llllrr}
\hline Radioisotope & $\begin{array}{l}\mathrm{E}_{\gamma} \\
(\mathrm{MeV})\end{array}$ & $\begin{array}{l}\text { Exp. } \\
\text { (Byun } \text { et al., 2004) }\end{array}$ & $\begin{array}{l}\text { M.C. } \\
\text { (Byun } \text { et al., 2004) }\end{array}$ & $\begin{array}{l}\text { Present } \\
\text { work }\end{array}$ & $\Delta_{1} \%$ \\
\hline${ }^{22} \mathrm{Na}$ & 0.511 & $0.912 \pm 0.002$ & $0.911 \pm 0.001$ & 0.901 & -1.221 \\
${ }^{137} \mathrm{Cs}$ & 0.662 & $0.947 \pm 0.028$ & $0.896 \pm 0.001$ & 0.890 & -6.404 \\
${ }^{60} \mathrm{Co}$ & 1.173 & $0.861 \pm 0.003$ & $0.845 \pm 0.001$ & 0.867 & 0.692 \\
${ }^{22} \mathrm{Na}$ & 1.275 & $0.857 \pm 0.003$ & $0.837 \pm 0.001$ & 0.860 & 0.349 \\
${ }^{60} \mathrm{Co}$ & 1.332 & $0.854 \pm 0.004$ & $0.832 \pm 0.001$ & 0.858 & 0.466 \\
\hline
\end{tabular}

Table 2. Comparison between relative total efficiencies of $0.511 \mathrm{MeV}{ }^{22} \mathrm{Na}$ axial point source placed at different positions inside the square hole of the parallelepiped detector.

\begin{tabular}{lllll}
\hline $\begin{array}{l}h \\
(\mathrm{~cm})\end{array}$ & $\begin{array}{l}\text { Relative total } \\
\text { efficiency } \\
\text { (Byun } \text { et } \text { al. },\end{array}$ & $\begin{array}{l}\text { Total } \\
\text { efficiency } \\
\text { [present } \\
\text { work] }\end{array}$ & $\begin{array}{l}\text { Relative total } \\
\text { efficiency } \\
\text { [present } \\
\text { work] }\end{array}$ & $\Delta_{2} \%$ \\
\hline 0 & 1 & 0.901 & 1 & 0 \\
2 & $0.997 \pm 0.01$ & 0.900 & 0.998 & 0.100 \\
4 & $0.990 \pm 0.01$ & 0.897 & 0.995 & 0.503 \\
6 & $0.977 \pm 0.01$ & 0.887 & 0.984 & 0.711 \\
8 & $0.962 \pm 0.01$ & 0.877 & 0.973 & 1.131 \\
10 & $0.936 \pm 0.01$ & 0.857 & 0.951 & 1.577 \\
\hline
\end{tabular}

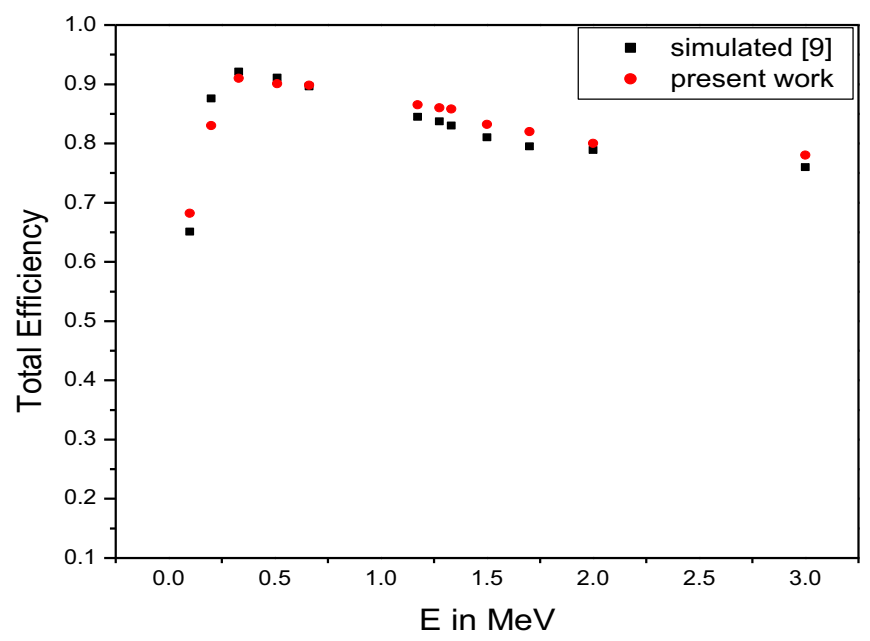

Fig. 3. Calculated (present work) and simulated total efficiencies for an isotropic radiating point source placed at the center of the detector $(h=0)$ as a function of the photon energy.

The calculated total efficiency (Eq. (2)) of the parallelepiped detector for different energy axial and non-axial point sources as a function of the source height $h$ are shown in Figures 4 and 5 .

The calculated total efficiency of the parallelepiped detector for disk and cylindrical sources is determined at different energies and heights using equations (31) and (32)

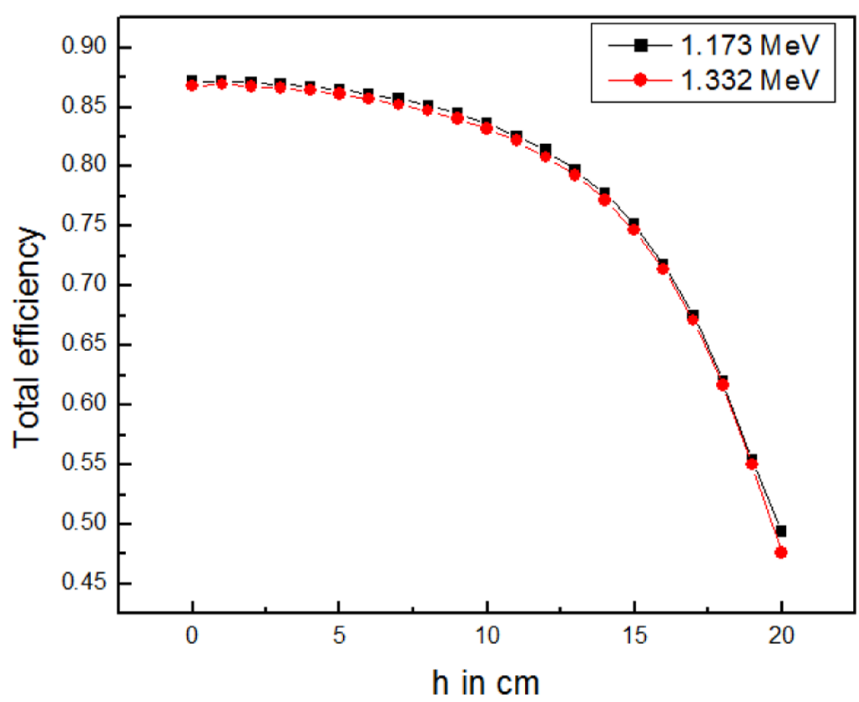

Fig. 4. Total efficiency as a function of the source-to-detector center distance $h$, in the case of an isotropic radiating axial point source $P(0, h)$ placed inside the detector hole.

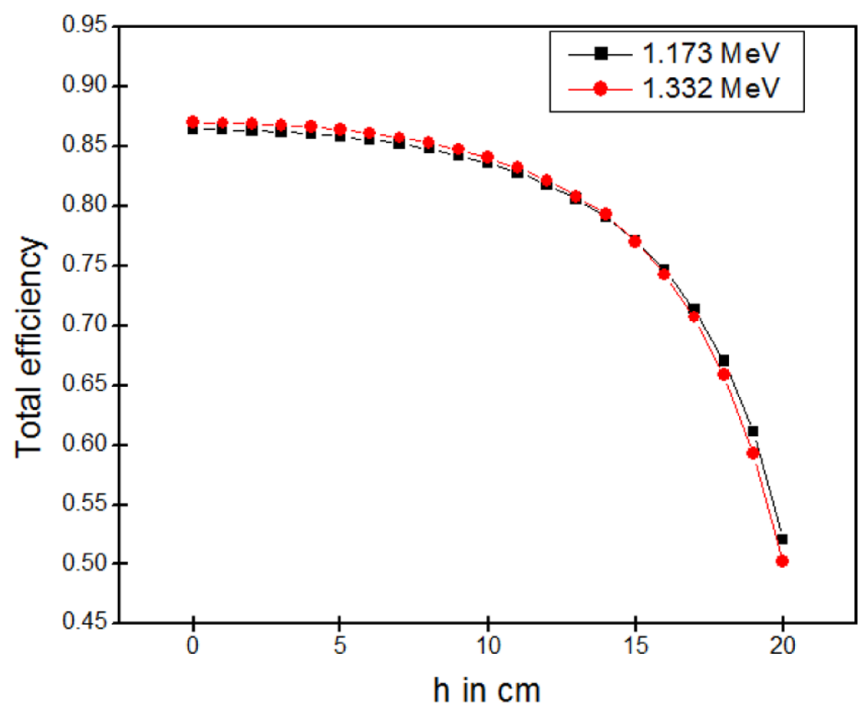

Fig. 5. Total efficiency as a function of the source-to-detector center distance $h$, in the case of an isotropic radiating non-axial point source $P(\rho, h)$ placed inside the detector hole. 


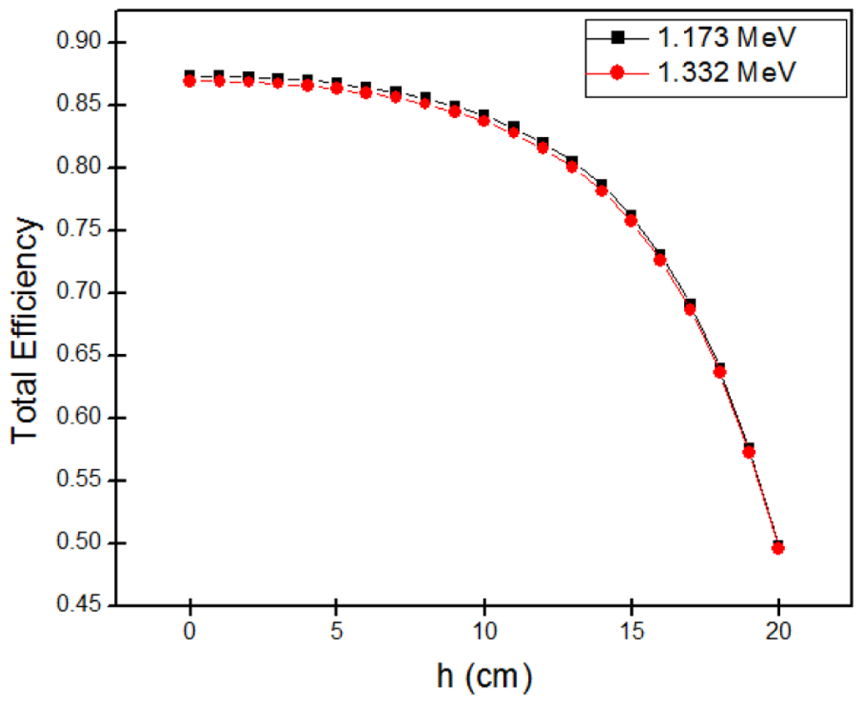

Fig. 6. Total efficiency as a function of the source-to-detector center distance $h$, in the case of disc source of radius $(s=5 \mathrm{~cm})$.

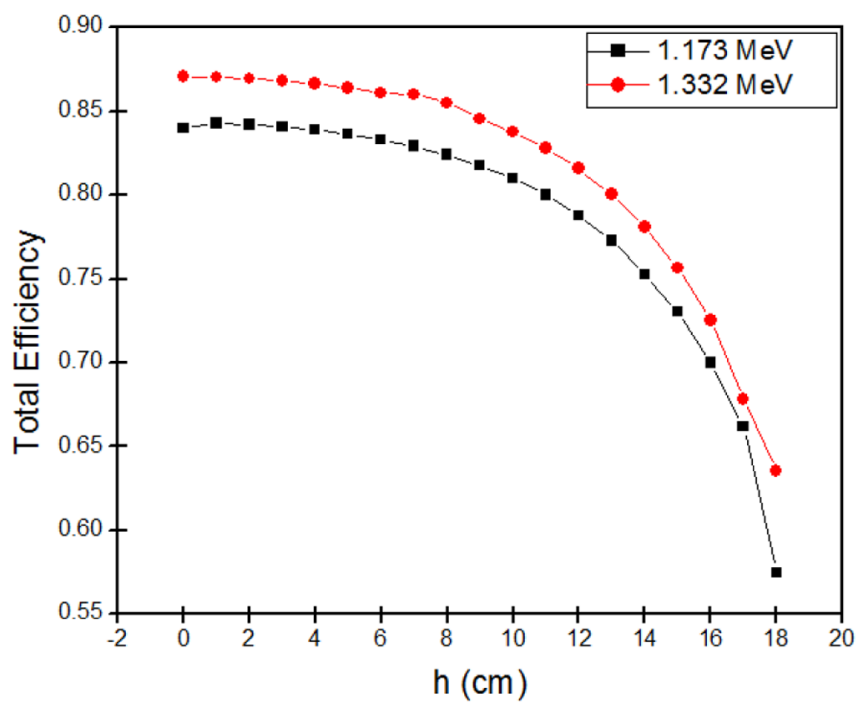

Fig. 7. Total efficiency as a function of the source-to-detector center distance $h$, in the case of cylindrical source of radius $(s=4 \mathrm{~cm})$ and height $(h=5 \mathrm{~cm})$.

respectively. The results shown in Figures 6 and 7 show also a decrease in total efficiency with the increase in the axial distance from the center of the detector. As it was mentioned before, total efficiency changes little in the central region of the detector

The average path length for an axial isotropic radiating point source at different positions is determined using equation (11) and the results are shown in Figure 8.

\section{Discussions}

The comparisons between the present work with experimental and Monte Carlo method (Byun et al., 2004) in Table 1 show a good agreement with maximum discrepancies of $6.4 \%$ in case of experimental and $2.7 \%$ in case of Monte Carlo. Since

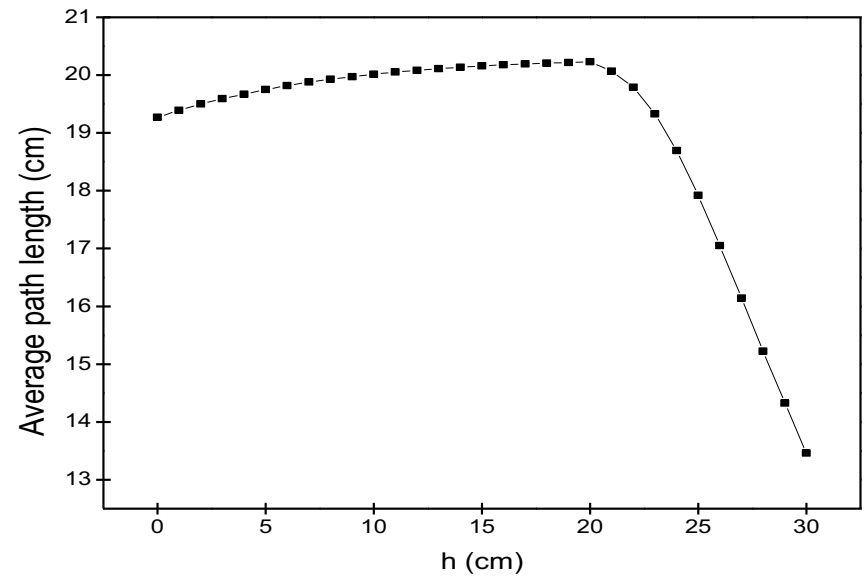

Fig. 8. The calculated average path length of the photon inside the parallelepiped detector at different axial positions from the center of the detector.

the discrepancies are less than $10 \%$, this indicates the validity of the present method. Moreover, it is much better than that of M.C, since no adjustment of the geometrical parameters of the used detector was applied and can be applied at low energies. Another comparison is also performed by comparing the relative total efficiencies at different heights as shown in Table 2. The results indicate also the validity of this method with maximum discrepancy of $1.6 \%$. Moreover, Figure 3 shows a good agreement between the simulated and the present work for different energies when the point source is placed at the center of the detector.

Since the discrepancies are small confirming the validity of our work, the study is extended to determine the total efficiency at different heights inside the detector for different source shapes as shown in Figures 4-7. Due to the enclosing geometry of the detection, the total efficiency changes a little when the source position is moved in the central region between 0 and $5 \mathrm{~cm}$ compared to the height of the detector $(40.6 \mathrm{~cm})$. This is also confirmed in Table 2 for ${ }^{22} \mathrm{Na}$ point source. In addition, the total efficiency decreased by $44 \%$ as the distance $h$ changes from 0 (center of the detector) to $20.3 \mathrm{~cm}$ (upper surface of the detector). This is due to the fact that not all the photons emitted from the source are recorded by the detector when it comes near to the surface. Moreover, the average path length shown in Figure 8 increases a little in the region between the detector central part and its surface. This is due to a small increase in the photon path lengths $d_{1}$ and $d_{2}$ in equation (11) and a slight decrease in the solid angle $\Omega$. In addition, when the source becomes out of the detector hole along the axial position, there is a rapid decrease in the average path length. This rapid decrease can be attributed to the sharp decrease in the path length $d_{1}$, and polar angles $\theta_{1}$ and $\theta_{2}$. In this case, the integration of $d_{1}$ in equation (11) approaches zero. Also, the path length $d_{2}$ decreases also as the distance of the source from the detector increases.

\section{Conclusions}

The numerical analytical method, which was established in the current work gives authorization for calculating the geometrical and the total efficiency of parallelepiped $\mathrm{NaI}(\mathrm{Tl})$ 
scintillation detector using several source geometries. The method is considered to be a simple formula for the calculation process, in particular for extended sources, like disk and cylindrical sources. In the comparisons of the efficiency values in Tables 1 and 2, the discrepancies between the present theoretical method with experimental results (in the case of the point source) and simulated method are less than $6.5 \%$ as in all published researches using this method. Since good agreement is obtained between experimental and theoretical results, the study is extended to determine the total efficiencies of nonaxial, disc and cylindrical sources at different energies and different distances from the center of the detector. In addition, the average path length of the parallelepiped detector is determined at different axil positions from the center of the detector.

\section{References}

Abbas M. 2001a. Analytical formulae for well-type $\mathrm{NaI}$ (Tl) and HPGe detectors efficiency computation, Appl. Radiat. Isot. 55: $245-252$.

Abbas M. 2001b. A direct mathematical method to calculate the efficiencies of a parallelepiped detector for an arbitrarily positioned point source, Radiat. Phys. Chem. 60: 3-9.

Abbas M. 2006. Analytical calculations of the solid angles subtended by a well-type detector at point and extended circular sources, Appl. Radiat. Isot. 64: 1048-1056.

Abbas M, Noureddeen S. 2011. Analytical expression to calculate total and full-energy peak efficiencies for cylindrical phoswich and lanthanum bromide scintillation detector, Radiat. Meas. 46: 440-445.

Al-Arbawy H. 2014. Study The effect of the shields movement and its thickness in detection efficiency by using scintillation detector $\mathrm{NaI}$ (Tl), Adv. Phys. Theor. Appl. 31: 53-57.
Baba H et al. 1991. Systematic treatment of the photopeak efficiency, Nucl. Instr. Meth. Phys. Res. A 309: 236-247.

Badawi M, El-Khatib A, Krar M. 2013. New numerical simulation approach to calibrate the $\mathrm{NaI}(\mathrm{Tl})$ detectors array using non-axial extended spherical sources, J. Instrum. 8: 11-18.

Byun SH et al. 2004. Efficiency calibration and coincidence summing correction for a $4 \pi \mathrm{NaI}(\mathrm{Tl})$ detector array, Nucl. Instr. Meth. Phys. Res. 535: 674-685.

Cejnar F, Kovář I. 1980. Experimental and calculated photopeak efficiency values for well-type $\mathrm{NaI}(\mathrm{Tl})$ detector, Int. J. Appl. Radiat. Isot. 31: 79-84.

Chatani H. 1999. Systematization of efficiency correction for gammaray disk sources with semiconductor detectors, Nucl. Instr. Meth. Phys. Res. 425: 291-301.

Gouda M, Badawi M, El-Khatib A, Abbas M. 2015. Calibration of well-type $\mathrm{NaI}(\mathrm{Tl})$ detector using a point sources measured out the detector well at different axial distances, J. Instrum. 10: 3-8.

Jiang SH et al. 1998. A hybrid method for calculating absolute peak efficiency of germanium detectors, Nucl. Instr. Meth. Phys. Res. 413: 281-292.

Kis Z et al. 1998. Comparison of efficiency functions for Ge gammaray detectors in a wide energy range, Nucl. Instr. Meth. Phys. Res. 418: 374-386.

Nafee S, Badawi M, Abdel-Moneim A, Abbas M. 2010. Calibration of the $4 \pi \gamma$-ray spectrometer using a new numerical simulation approach, Appl. Radiat. Isot. 68: 1746-1753.

National Institute of Standard and Technology, Physical Measurements Laboratory. 1998. XCOM Photon Cross-Sections Database, http://physics.nist.gov/PhysRefData/Xcom/html/xcom1.html.

Sánchez-Reyes AF, Febrián MI, Baró J, Tejada J. 1987. Absolute efficiency calibration function for the energy range $63-3054 \mathrm{keV}$ for a coaxial Ge(Li) detector, Nucl. Instr. Meth. Phys. Res. B 28: 123-127.

Yücel H, Cetiner MA, Demirel H. 1996. Dependence of photon interaction depth on linear attenuation coefficient in high pure germanium detectors, Appl. Radiat. Isot. 47: 535-541.

Cite this article as: Noureddeen SF, Abbas MI. 2017. New numerical evaluation of parallelepiped NaI(Tl) detector geometrical and total efficiencies using extended sources. Radioprotection 52(4): 265-271 